\title{
Localization of Plasmons in Self-assembled Doped-semiconductor Nanocrystal Arrays
}

Kevin Roccapriore $^{1}$, Shin $\mathrm{Cho}^{2}$, Delia Milliron ${ }^{2}$ and Jordan Hachtel ${ }^{1}$

${ }^{1}$ Oak Ridge National Laboratory, Oak Ridge, Tennessee, United States, ${ }^{2}$ The University of Texas at Austin, Austin, Texas, United States

Noble metals, such as silver and gold, are the archetypal materials used in plasmonic structures. However, there are many applications that require telecommunication wavelengths or lower and, in general, noble metals do not provide the necessary spectral behavior at these frequencies. It was quickly realized that doping semiconductors can allow enough conduction electrons to participate in a collective response in NIR and lower energies ${ }^{1,2}$, even with comparable quality factors to silver and gold. Moreover, these colloidally prepared nanocrystals can be coated with ligands and promote self-assembled growth of periodic nanostructures.

Here, we examine fluorine-doped indium tin oxide (F:ITO) nanoparticles of $~ 10-20 \mathrm{~nm}$ dimensions that have self-assembled into a hexagonal lattice. It is well-known that when several plasmonic nanocrystals come into close proximity, their plasmon modes can hybridize, leading to modes not seen in single particles. Recent advances in monochromation technology ${ }^{3}$ has allowed electron energy loss spectroscopy (EELS) in a scanning transmission electron microscope (STEM) to detect excitations in the NIR and even lower, while retaining the resolution to spatially distinguish between different plasmon modes directly at the nanoscale.

Arranging plasmonic nanocrystals into a much larger array has a similar effect of plasmon hybridization, however the added complexity of the large-scale arrays results in some interesting differences from the standard hybridization picture. One of the key differences is the presence of heterogeneity in the selfassembled film. Ordinarily, plasmons probed by an electron are not observed more than roughly a few nanometers from the object surface. In contrast, at the edges or near breaks in the self-assembled film, a highly delocalized array plasmon mode is observed that is strongly excited even from several hundreds of nanometers from the edge of the array.

The delocalization of the array plasmon mode with respect to the other modes observed in the array is illustrated in Figure 1. Here, a spectrum image is acquired over a large break in the monolayer film (dark field STEM image shown in left panel). A four Lorentzian peak fit is performed on the spectrum image to produce two-dimensional maps of the plasmon peaks (the color bar corresponds to the peak frequency, while the color intensity corresponds to the excitation strength). The array plasmon is shown in peak (I) which is the dominant mode in the plasmonic response. The delocalization of the mode can be clearly seen not directly at the edge of the array but even deep into the break (towards the top of the image). The critical difference is clear by comparing the delocalization (and edge-redshift) of the array mode compared to that seen in plasmon modes II, III, and IV, which are spatially localized to the particles and do not change in energy, which is expected behavior of LSPRs. 

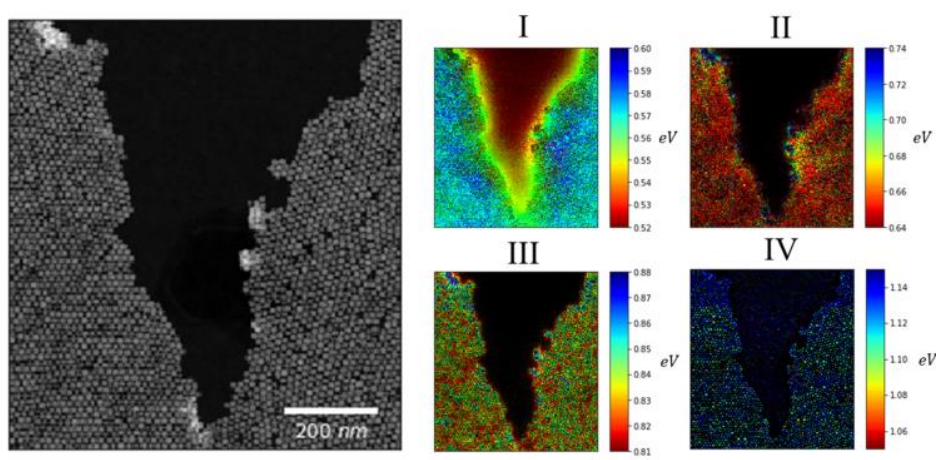

IV

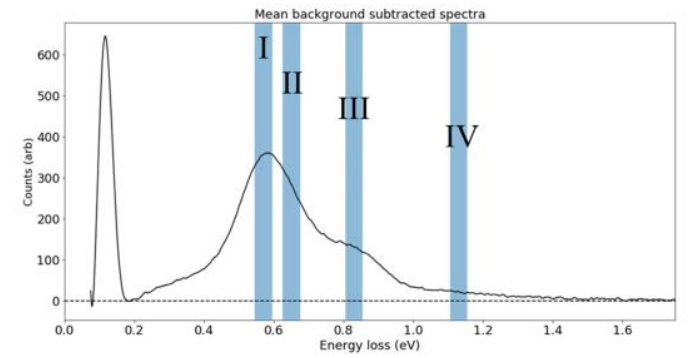

Figure 1. The non-local array-supported plasmon mode is evident in hyperspectral map (I). HAADF structural map (upper left) and spectral maps obtained by Lorentzian peak fitting (upper right). The color levels refer to peak energy, while the intensity refers to excitation strength. The average spectra of the entire spectrum image and center peak positions are shown (bottom).

References

1. Stanley, R. Plasmonics in the mid-infrared. Nat. Photonics 6, 409-411 (2012).

2. Agrawal, A. et al. Localized Surface Plasmon Resonance in Semiconductor Nanocrystals. Chem. Rev. 118, 3121-3207 (2018).

3. Krivanek, O. L. et al. High-energy-resolution monochromator for aberration-corrected scanning transmission electron microscopy/electron energy-loss spectroscopy. Philos. Trans. R. Soc. Math. Phys. Eng. Sci. 367, 3683-3697 (2009).

Acknowledgements

This effort is based upon work supported by the U.S. Department of Energy (DOE), Office of Science, Basic Energy Sciences (BES), Materials Sciences and Engineering Division and was performed at the Oak Ridge National Laboratory's Center for Nanophase Materials Sciences (CNMS), a U.S. Department of Energy, Office of Science User Facility. This research was conducted, in part, using instrumentation within ORNL's Materials Characterization Core provided by UT-Battelle, LLC, under contract No. DEAC05-00OR22725 with the DOE 